\title{
A cross sectional comparison of postnatal care quality in facilities participating in a maternal health voucher program versus non-voucher facilities in Kenya
}

Charlotte E Warren ${ }^{1}$, Timothy Abuya ${ }^{2 *}$, Lucy Kanya ${ }^{2}$, Francis Obare ${ }^{2}$, Rebecca Njuki ${ }^{2}$, Marleen Temmerman ${ }^{3}$ and Ben Bellows ${ }^{2}$

\begin{abstract}
Background: Health service fees constitute substantial barriers for women seeking childbirth and postnatal care. In an effort to reduce health inequities, the government of Kenya in 2006 introduced the output-based approach (OBA), or voucher programme, to increase poor women's access to quality Safe Motherhood services including postnatal care. To help improve service quality, OBA programmes purchase services on behalf of the poor and marginalised, with provider reimbursements for verified services. Kenya's programme accredited health facilities in three districts as well as in two informal Nairobi settlements.

Methods: Postnatal care quality in voucher health facilities $(n=21)$ accredited in 2006 and in similar non-voucher health facilities $(n=20)$ are compared with cross sectional data collected in 2010. Summary scores for quality were calculated as additive sums of specific aspects of each attribute (structure, process, outcome). Measures of effect were assessed in a linear regression model accounting for clustering at facility level. Data were analysed using Stata 11.0.

Results: The overall quality of postnatal care is poor in voucher and non-voucher facilities, but many facilities demonstrated 'readiness' for postnatal care (structural attributes: infrastructure, equipment, supplies, staffing, training) indicated by high scores (83/111), with public voucher facilities scoring higher than public non-voucher facilities. The two groups of facilities evinced no significant differences in postnatal care mean process scores: 14.2/ 55 in voucher facilities versus $16.4 / 55$ in non-voucher facilities; coefficient: $-1.70(-4.9,1.5), p=0.294$. Significantly more newborns were seen within 48 hours ( $83.5 \%$ versus $72.1 \%: p=0.001$ ) and received Bacillus Calmette-Guerin (BCG) $(82.5 \%$ versus $76.5 \%$ : $p<0.001)$ at voucher facilities than at non-voucher facilities.

Conclusions: Four years after facility accreditation in Kenya, scores for postnatal care quality are low in all facilities, even those with Safe Motherhood vouchers. We recommend the Kenya OBA programme review its Safe Motherhood reimbursement package and draw lessons from supply side results-based financing initiatives, to improve postnatal care quality.
\end{abstract}

Keywords: Postnatal care, Vvoucher, OBA, Quality of care, Structure, Process and outcome

\footnotetext{
* Correspondence: tabuya@popcouncil.org

${ }^{2}$ Population Council General Accident Insurance House, Ralph Bunche Road, PO Box 17643, Nairobi 00500, Kenya

Full list of author information is available at the end of the article
} 


\section{Background}

An estimated $60 \%$ of the world's 270,000 annual maternal deaths occur within 48 hours of delivery, and two thirds of the three million annual newborn deaths occur within the first week $[1,2]$. Early treatment in the postnatal period could save more lives and support adoption of healthy behaviours [3]. The postnatal period is largely neglected, however, and women and infants rarely receive optimal postnatal care [4].

Poverty and inequity are two primary underlying causes of maternal and newborn deaths in low and middle income countries [5]. Formal and informal fees for provision of healthcare during pregnancy, childbirth, and the immediate postnatal period constitute a substantial financial barrier for poor women [6-8]. To address these challenges, governments and donors have been prompted to find innovative ways of increasing access to maternal and newborn health services such as subsidised consumer led demand for quality services [9-12]. Vouchers and strategies incorporating supply and demand components, like the output-based approach (OBA), have demonstrated increases in service utilisation among targeted populations [6, 9, 11, 13-16].

A primary OBA or voucher programme objective is the increase of service utilisation by improving high priority populations' purchasing powers, with a secondary objective of improving quality through reimbursements to providers for verified service delivery. Service reimbursements link pre-defined quality or quantity indicators to financial reimbursements, and in concert with competition with other providers or health facilities (public or private), motivate improvements in equitable access and quality of maternal and newborn health services including postnatal care [13, 17]. Other resultsbased or performance-based financing strategies focus on incentives for quality improvements that may not accelerate uptake of services among the poor and marginalised $[18,19]$.

Kenya's maternal mortality ratio and newborn mortality rate are 488/100,000 live births and 31/1000 live births, respectively [20]. Over $90 \%$ of women attend antenatal care at least once, $44 \%$ give birth with a skilled attendant, and $47 \%$ receive postnatal care [20]. Of the women who receive postnatal care, $42 \%$ said they received a health check during the first two days, but no data provide information on the content of their postnatal care. Higher order births, and those in rural areas, are less likely to receive postnatal care than those of lower order and in urban areas. Generally, the most important characteristics of women associated with postnatal care are higher wealth statuses and having received antenatal care $[20,21]$. Results from the 2010 Kenya service provision assessment indicate that only $59 \%$ of facilities (from 34 \% in the 2004 assessment) offer postnatal care services (compared to $74 \%$ offering antenatal care). Although targeted postnatal care has reputedly increased emphasis on the first 48 hours after birth and includes check-ups through the first year, no specific module for postnatal care exists in the service provision assessment besides information in the antenatal care module [22].

\section{Financing strategy to improve quality of maternal and newborn health services in Kenya}

The OBA project is intended to improve both the quality and access of appropriate maternal and newborn health services for the poor, through subsidized service purchase contracts with public and private providers [23-25]. Sold at highly subsidized prices, these vouchers provide direct subsidies to the poorest populations, permitting their access to Safe Motherhood services, and ultimately increasing their uptake, with qualified and certified service providers providing the services. The Ministry of Health $(\mathrm{MoH})$ oversees implementation, supported by both the German Development Bank (KfW) and the Government of Kenya, with daily management, of voucher distribution, health facility claims and reimbursement, by a contracted private party as the voucher management agency [11, 13]. Criteria for accrediting public, private, and faith-based facilities were developed by the OBA project, adapted from existing national standards and guidelines, that are reviewed annually [23, 24].

Accredited health facilities are reimbursed for services for each voucher holder. The OBA programme provides participating facilities only with training for voucher reimbursement with the voucher management agency. In theory, health managers then use their disbursed funds to improve their facilities for a competitive advantage in encouraging use of their services (by both voucher holders and the general population) by procuring additional equipment, medicines, and supplies, along with building extensions and improving existing buildings, water sources, electricity, and other services, and updating their health care providers' essential obstetric and newborn skills [26].

The voucher's primary beneficiaries are economically disadvantaged women of reproductive age from Kisumu, Kitui, and Kiambu counties and two informal settlements in Nairobi County (Korogocho and Viwandani). Community distributors appointed by the voucher management agency sell the Safe Motherhood vouchers at a subsidised cost of 200 Kenyan shillings (equivalent to US\$2.50). To identify poor women who qualify, distributors use a poverty grading tool assessing eight dimensions of household assets and amenities, expenditures and income, and health service access that are unique for each county. Any woman scoring eight to 16 points 
qualifies for the voucher. The design of the overall Kenya OBA voucher program is described in detail elsewhere $[23,24]$.

The Safe Motherhood voucher subsidises four antenatal visits, labour and delivery, and one postnatal visit. Safe Motherhood voucher clients are entitled to comprehensive essential obstetric and neonatal care including comprehensive newborn and post-delivery care $[13,23$, 27]. The voucher programme offers two other vouchers: one for long acting and permanent family planning methods and the other for gender-based violence recovery services, but these are not discussed in this paper $[11,28]$.

Although evidence suggests that voucher programmes (including the Kenya programme) have potential for improving both reproductive health service utilisation [6, $10,14]$ and quality of care $[15,16,29,30]$, none have specifically examined whether Safe Motherhood vouchers affect the quality of postnatal care, the most neglected element in the continuum of care. This paper attempts to fill that gap by comparing Kenya OBA programme health facilities, for four years, between 2006 and 2010, with a comparable cross-section of facilities without contracts for voucher clients ("non-voucher facilities"). This study hypothesizes that the quality of postnatal care in voucher facilities will be equal to or greater than the quality of those same services in nonvoucher facilities [31].

\section{Methods}

This paper uses data from a larger quasi-experimental design that evaluated the impact of the Kenyan OBA voucher scheme on selected reproductive health services' quality and access. The larger study compared voucheraccredited health facilities with non-voucher facilities in counties with similar characteristics at two points in time (in 2010 and 2012); its study methodology is described in detail elsewhere [31]. For our analysis we used cross sectional data from 2010 to compare quality of postnatal care in health facilities accredited by the voucher programme since its inception in 2006 with similar non-voucher health facilities (a "post-test design"). We compare key outcomes from quality of postnatal care with similar non-voucher facilities.

This design was chosen due to the lack of random assignment of health facilities within intervention (voucher) or comparison (non-voucher) sites [31] because voucher sites were identified by $\mathrm{MoH}$ and KfW based on service gaps and need for increasing service coverage and availability for low income and remote populations. Facilities in targeted counties were asked to participate and were contracted if they met the accreditation criteria. Population Council, in conjunction with $\mathrm{MoH}$, identified neighbouring counties with similar non- voucher facilities to maximise the likelihood of similar social, cultural, and economic characteristics in addition to similar reproductive health and healthcare behaviours among women 15 to 45 years old to serve as the comparison group [31]. Comparison facilities' characteristics were similar to voucher facilities in their type of practice, available professional skills, clientele, locations, fees, and services including their levels (hospital, nursing home or health centre) and ownership type (public, private, faith based or non-governmental) [31].

\section{Study procedures}

This paper uses quantitative data from health facility assessments in 41 health facilities (20 public and 21 private-for-profit or faith-based). The 21 voucher facilities were randomly selected from 56 accredited health facilities in the three counties (Kiambu, Kisumu, Kitui) and compared with 20 facilities from three non-voucher counties (Nyandarua, Uasin Gichu, Makueni). The health facility assessments used four study instruments:

Facility inventories All inventories included a checklist for availability of infrastructure, equipment, commodities, medicines and supplies, as well as number of staff and services provided, along with provider training for staff $(n=41)$.

Interviews with 163 maternal health care providers All maternal and newborn health providers working in facilities during data collection were approached for permission to interview them. Based on normal staffing levels, we expected four to eight providers eligible for interviewing in hospitals, and between two and four at health centres, for approximately 80 providers in each group and a total of 160. A total of 90 providers from voucher facilities and 73 in non-voucher facilities were interviewed. Providers were asked questions on their technical competence and time utilisation during the postnatal period.

Observations of 794 client-provider interactions Client-provider interaction includes both a consultation's process (how clients are treated and whether they actively participate) and content (what they are told, along with technical competence, accuracy of information and provision of essential information). Trained research assistants with clinical backgrounds observed postnatal consultations, assessing the consultation process with a standardized checklist. Subsequent sessions were observed, after patients' informed consent, for 18 postnatal care clients in each facility (a total of 360 expected). A total of 499 postnatal care consultations in voucher facilities and 295 in non-voucher facilities were observed. 
Fewer clients in non-voucher facilities attended during the data collection period.

Exit interviews with $\mathbf{7 2 8}$ postnatal clients Research assistants interviewed women as they exited their postnatal care consultations, focusing on the services, information, and counselling they received, as well as their fertility desires and postpartum family planning. A total of 484 exit interviews were conducted in voucher facilities, and 244 in non-voucher facilities.

\section{Ethics}

Ethical approval for the evaluation was granted by Population Council's Institutional Review Board (IRB) No. 470 and Kenya Medical Research Institute (KEMRI) SCC 174. All women attending PNC services during the data collection period were asked written permission to observe their consultation and to be interviewed afterwards. Written informed consent was obtained prior to all interviews that were conducted in settings that ensured privacy and confidentiality. Participants were informed they could withdraw from the research at any time. Data collectors were trained on ethical conduct.

\section{Quality of Care framework and development of scores}

A study definition and framework for quality of care was adapted from Donabedian and Bruce using three general elements of quality: structure, process, and outcome [32, 33]. The elements of quality of care were assessed using health facility inventories, provider interviews, clientprovider interactions, and client exit interviews (Table 1). Total scores were developed for the essential components of quality care. A list of minimum equipment required for postnatal care services, for example, was developed based on $\mathrm{MoH}$ guidelines [34, 35] and consultations with service providers: a working blood pressure machine; stethoscope; spotlight, flashlight or examination light; examination couch; baby weight scale; adult weight scale; and autoclave or other sterilizer.

To develop an overall quality score, a composite scoring system was generated by combining several indicators into a single score [36]. There are two methods for generating composite quality scores. The "Opportunity Model" is based on the percentage of functions ("quality indicators") actually performed compared to the total number of targeted functions [37]. If a total of 125 functions, of a targeted total of 250, were performed on 10 clients, then the composite Opportunity Model quality score would equal $0.5(=125 / 250)$. Typically, equal weighting is assumed for each function, which helps derive an aggregate composite quality score covering all functions. The second scoring method employs a predefined criteria system, the "Grading Model". For example, in Zambia a national assessment of the quality of antenatal care classified all health facilities into three grades, "optimum", "adequate", or "inadequate", using a set of criteria for access to care, responsiveness and appropriateness, continuity of care, patient safety, and effectiveness and efficiency [38]. We have used the Opportunity Model due to its ease of interpretation, with detailed scores for each function, group of functions, and aggregate. The Opportunity Model allows for easier comparison both within categories (e.g., indicators or groups of indicators) and among groups (e.g., facilities, sub-counties), whereas the Grading Model would make differentiation of two groups with the same grade difficult.

Table 1 Quality of Care Framework

\begin{tabular}{|c|c|c|}
\hline Structure & Process & Outcome \\
\hline $\begin{array}{l}\text { Data source: Facility } \\
\text { inventory and } \\
\text { providerknowledge }\end{array}$ & $\begin{array}{l}\text { Data source: } \\
\text { Observations of client - } \\
\text { provider interactions }\end{array}$ & $\begin{array}{l}\text { Data Source: Client } \\
\text { exit interviews and } \\
\text { service statistics }\end{array}$ \\
\hline Facility inputs & QoC & Reduction in \\
\hline $\begin{array}{l}\text { Appropriate } \\
\text { availability of services }\end{array}$ & - Quality of clinical care & - Waiting time \\
\hline - Facility readiness & $\begin{array}{l}\text { - Interpersonal care/ } \\
\text { rapport }\end{array}$ & Improvement in: \\
\hline - $24 \mathrm{~h}$ availability & - History taking & $\begin{array}{l}\text { - Interpersonal } \\
\text { relations }\end{array}$ \\
\hline $\begin{array}{l}\text { - Emergency } \\
\text { preparedness }\end{array}$ & $\begin{array}{l}\text { - Range of services } \\
\text { offered }\end{array}$ & $\begin{array}{l}\text { - Time spent with } \\
\text { provider }\end{array}$ \\
\hline $\begin{array}{l}\text { - Equipment, } \\
\text { medicine and } \\
\text { supplies }\end{array}$ & - Maternal health care & Client understanding \\
\hline -Infection prevention & - Infant health care & - Client choice: \\
\hline -Infrastructure & $\begin{array}{l}\text { - Danger signs for } \\
\text { mother and infant }\end{array}$ & $\begin{array}{l}\text { - RH goals and family } \\
\text { planning }\end{array}$ \\
\hline $\begin{array}{l}\text { - IEC materials } \\
\text { available }\end{array}$ & • Family planning & - Knowledge \\
\hline $\begin{array}{l}\text { - Guidelines and } \\
\text { registers }\end{array}$ & - HIV services & - Increase in: \\
\hline $\begin{array}{l}\text { Technical } \\
\text { Competence }\end{array}$ & $\begin{array}{l}\text { Information given to } \\
\text { client }\end{array}$ & - FP uptake \\
\hline $\begin{array}{l}\text {-Education and } \\
\text { Training }\end{array}$ & $\begin{array}{l}\text { - Assess client } \\
\text { understanding }\end{array}$ & $\begin{array}{l}\text { - Infant immunisation } \\
\text { uptake }\end{array}$ \\
\hline - Supervision & - Documentation & - Satisfaction \\
\hline \multirow[t]{6}{*}{ - Provider knowledge } & & $\begin{array}{l}\text { - Confidentiality/ } \\
\text { privacy }\end{array}$ \\
\hline & & $\begin{array}{l}\text { - Continuity of care/ } \\
\text { followup }\end{array}$ \\
\hline & & $\begin{array}{l}\text { Increase service } \\
\text { provision }\end{array}$ \\
\hline & & - Range of services \\
\hline & & - Multiple service use \\
\hline & & $\begin{array}{l}\text { - Diversification of } \\
\text { client profile }\end{array}$ \\
\hline
\end{tabular}


To measure the readiness of a facility to provide quality postnatal care, its attributes (infrastructure, equipment, commodities, drugs and supplies required for anyone to provide comprehensive postnatal care) are added, with equal weights, to create a structural score with a maximum of 107 points. The facility inventory study tool recorded these scores as well as each facility's human resource elements including staffing numbers and availability of appropriate services. An additional scoring section, with a total of 32, assessed provider knowledge and training on maternal and newborn healthcare and postpartum family planning (including technical updates), with data drawn from provider interviews. The provider score was incorporated into the facility's total score, for a potential composite score of 139 per facility.

Process attributes for quality, based on national and international standards [34, 39, 40] were analysed using data from our observations of client-provider interactions during postnatal consultations. Using the methodology described facility scores, summary process attributes for technical competence with equal weights (total score of 47) were derived from the observation checklists of client-provider interactions. These observations included how a provider performed in history taking, physical examinations of mother and baby, and counselling on maternal and newborn danger signs, return to fertility and healthy timing and spacing of pregnancies, infant feeding, and HIV and STI risk assessment and management, as well as consultation documentation. Interpersonal relations (with a score between 0 and 8) including privacy, confidentiality, and rapport between client and provider were also captured (Table 2).

Outcome attributes focus on postnatal clients' experiences and perceptions of their quality of care, including waiting times, perceptions of respect accorded, their understanding and knowledge of their health statuses, whether they asked questions during their consultations, opportunity to ask questions, range of services received for both mother and baby, and mechanisms encouraging follow up appointments (Table 2).

\section{Data analysis}

Quantitative data were double entered using Epidata and exported to Stata 11 for analysis. For each component of quality of care-structure, process and outcome-summary scores were calculated as the sum of items representing specific aspects of each attribute (as defined above), and these demonstrate the overall quality score. Two-tailed unpaired t-tests with unequal variance evaluated group differences in the average process scores comparing intervention and comparison groups. A pvalue of less than or equal to 0.05 was the threshold for significance. Pearson's chi-square tests were used to evaluate differences in proportions for various patientreported outcome measures that included waiting times to see providers, time spent with providers, and patients reporting satisfaction.

To assess measures of effect, a linear regression model was used for individual and summative process quality score outcomes. We controlled for clustering at facility level, type of provider defined as public or private, and level of care (hospitals and sub-district hospitals versus dispensaries, nursing homes and clinics). In all instances we report coefficients with their $95 \%$ confidence intervals and $p$ values for the coefficients for all three models.

\section{Results}

\section{Characteristics of women attending postnatal care}

The average ages (25.6 years) of postnatal women observed and interviewed were similar at voucher $(n=451)$ and non-voucher facilities $(n=237)$. There were no signifcant differences $[p=0.327]$ in education between the two groups, with most women reporting completion of primary $(51.9 \%)$ or secondary school (31.6\%). Around $82.2 \%$ of women attending voucher facilities were married, compared to $87 \%$ of women at non-voucher facilities $[p=0.078]$ (data not shown). There were no signficant differences between the poorest women (bottom two quintiles) attending voucher facilities and nonvoucher facilities (32.6\% versus $39.3 \%$; $p=0.077$ ). Significantly more women at voucher facilities said they would find it very difficult (31.3\% versus $9.5 \%$ ) or difficult (50.1\% versus $45.7 \%$ ) than women at non-voucher facilities to pay a health bill of more than 1,000 Kshs (US\$12) $[p<0.001]$ (data not shown in tables).

Fifty seven percent of voucher facility clients used a Safe Motherhood voucher. The two primary reasons for women's attendance for postnatal services were care for themselves and immunisations for their babies. Significantly more women at voucher facilities attended for infant immunisations than woman at non-voucher facilities $(64.4 \%$ versus $60.7 \% ; p<0.001)$, with fewer attending for postnatal care services (19.5\% versus $25.6 \%$; $p<0.001)$.

\section{Characteristics of health facilities}

Facilities assessed are described in Table 3. The staffing cadres in the two facility groups were similar besides the fact that more medical doctors were available at voucher facilities. Postnatal consultations were generally performed by two cadres of nurses. There were no significant differences $(p=0.503)$ for registered nurses or midwives conducting postnatal care consultations at voucher facilities (68.0\%) and non-voucher facilities (61.3\%), but significantly fewer enrolled nurses or 
Table 2 Attributes of care: structure, process and outcome

\begin{tabular}{|c|c|}
\hline Attributes of quality & Elements assessed \\
\hline \multicolumn{2}{|c|}{ Structure attributes: Infrastructure equipment and supplies (0-107) } \\
\hline $\begin{array}{l}\text { FP commodities available (score } \\
\text { from 0-11) }\end{array}$ & $\begin{array}{l}\text { Combined pill, Progestin only pill, Emergency Contraceptives, Injectables, IUCD, implants, male and female } \\
\text { condoms, male and female sterilization, fertility based methods. }\end{array}$ \\
\hline Testing reagents available (0-12) & $\begin{array}{l}\text { Reagents for HIV (Elisa HIV-1 and Elisa HIV-2) Rapid reagents for HIV testing, Reagents for anaemia test, TB, urin- } \\
\text { ary tract infections, malaria, cervical cancer screening and pregnancy test }\end{array}$ \\
\hline General supplies (0-6) & $\begin{array}{l}\text { Disposable needles and syringes, Insecticide treated nets, specimen bottles/pots for urine, sputum and blood, } \\
\text { slides for malaria parasites }\end{array}$ \\
\hline Essential vaccines (0-6) & Tetanus Toxoid, BCG, Measles, Polio, Hepatitis B, Pentavalent \\
\hline ARV Drugs (0-7) & $\begin{array}{l}\text { Nevirapine tabs and syrup, Zidovudine (ZDV, AZT),AZT syrup, Stavudine, Zidovudine + Lamivudine } \\
\text { (Combivir),Miconazole or clotrimazole pessaries, }\end{array}$ \\
\hline STI and RH drugs (0-10) & $\begin{array}{l}\text { Ciprofloxacin oral, Erythromycin oral, Tetracycline oral, Benzathine Penicillin, Cotrimoxazole tabs and syrup, } \\
\text { Metronidazole tablets, Metronidazole IV, Gentamicin IV, Amoxycillin }\end{array}$ \\
\hline Infection control supplies (0-9) & $\begin{array}{l}\text { Sterile and clean latex gloves, clean non-latex gloves, decontamination solution, waste receptacle with and } \\
\text { without lid and plastic liner, container for used sharps, single use hand drying towels or a functioning electric } \\
\text { hand dryer, running water }\end{array}$ \\
\hline FP equipment/supplies (0-18) & $\begin{array}{l}\text { speculum (small/medium/ large), tenacula, troca, surgical scissors, kidney dishes, sponge holding forceps, } \\
\text { mosquito forceps - curvedand straight, surgical blade: size 15/11, draping towels, betadine, gauze, elastoplast }\end{array}$ \\
\hline Delivery supplies/kits (0-5) & Delivery kit, Suture kit, Minilap BTL kit, Foetal scope, MVA kit \\
\hline General equipment $(0-7)$ & $\begin{array}{l}\text { A working blood pressure machine, stethoscope, spotlight or flashlight orexamination light, examination } \\
\text { couch, functional weighing scales for babiesand adults, autoclave/ sterilizer }\end{array}$ \\
\hline Emergency equipment (0-5) & Oxygen, Adult and newborn resuscitation set Magnesium Sulphate, Calcium gluconate. \\
\hline General infrastructure/utilities (0-11) & $\begin{array}{l}\text { Waiting area is shaded and with seats, Waiting area for new admissions, Heater for delivery room and nursery, } \\
\text { Functioning delivery bed, Postnatal ward, Private space for FP, ANC and PNC examination, } 24 \text { h supply of } \\
\text { clean water and power to ensure fridge remains functional, Reliable lighting, Client toilets, Clean water for } \\
\text { drinking with clean cups/glasses. }\end{array}$ \\
\hline
\end{tabular}

Structure attributes: Provider knowledge and training (0-32)

Updates in last 24 months (0-7) Family planning, contraceptive technology updates, targeted postnatal care, PMTCT, screening for cervical cancer, EONC.

Knowledge of postnatal care (0-5) Routine health care for mother and baby, return to fertility, family planning counselling, infant feeding, immunizations

Knowledge of maternal danger signs Foul smelling vaginal discharge, heavy vaginal bleeding, severe lower abdominal pain, fever with or without $(0-7)$ chills, swollen hands, face, legs, severe headache and/or blurred vision. excessive tiredness or breathlessness,

Knowledge of basic preventive newborn care $(0-7)$

Early initiation of and exclusive breastfeeding for 6 months, early detection of problems/anger signs, clean delivery practices, warmth, cord, eye care.

Knowledge of danger signs in Poor or no breastfeeding /feeding, difficulty breathing, hypothermia or hyperthermia, septic spots/boils on newborn (0-6) body, restlessness or irritability, jaundice.

Process attributes: Provider technical skills (0-47)

History taking (0-7)

Physical examination (0-7)

Danger signs advice given (0-3)

Fertility advice given (0-5)

STI/HIV risk assessment (0-3)

STI/HIV risk factors (0-4)

STI management (0-3)

Infant feeding advice (0-3)

Infant examination (0-4)
Date of delivery, if resumed menses, about HIV status, about medication currently taken, place of delivery, mode of delivery, if currently breast feeding.

Took client's temperature and blood pressure, check for pallor (anaemia), examine breasts and nipples, palpate the client's abdomen for uterine involution, checked perineum and discharge/lochia, checked extent of PV bleeding.

Excessive vaginal bleeding, fever with or without chills, broken scars(Perineum/Caesarean).

Discuss return to fertility, discuss healthy timing and spacing of pregnancies, discuss the health benefits for mother and baby when birth spacing, resume sexual activity and discussion of any method.

STI with the client, HIV/AIDS with the client, STI and/or HIV risk factors with the client.

Multiple partners, STIs increase risk of HIV, unprotected sexual intercourse, not knowing partner's status.

Give information on symptoms of an STI, screen for STI, advise to seek medical treatment if they notice STI symptoms.

Discussed infant feeding, discuss how mother was managing with breastfeeding, re-emphasize exclusive feeding (either breast or replacement).

Examine baby (undressed), check temperature, check baby's respirations, baby weighing. 
Table 2 Attributes of care: structure, process and outcome (Continued)

\begin{tabular}{|c|c|}
\hline Infant danger signs discussed (0-4) & Feeding difficulties - not sucking or sucking poorly, breathing difficulties, body feels hot or too cold, jaundice. \\
\hline Documentation (0-4) & $\begin{array}{l}\text { Provider looked at client's health card before beginning the consultation/ while collecting information/ } \\
\text { examining the client, has a post-partum register, recorded information in register/tally sheet wrote on the cli- } \\
\text { ent's card. }\end{array}$ \\
\hline \multicolumn{2}{|c|}{ Process attributes: Provider interpersonal skills(0-7) } \\
\hline Rapport $(0-7)$ & $\begin{array}{l}\text { Greets client, used clients name, introduces herself, tells client what will be done, Encourages client to ask } \\
\text { question, ensured privacy, assures confidentiality, courteous to client throughout. }\end{array}$ \\
\hline \multicolumn{2}{|c|}{ Outcome attributes: Client experiences and services received } \\
\hline $\begin{array}{l}\text { Family planning uptake within 0-10 } \\
\text { weeks }\end{array}$ & Proportion of clients receiving preferred methods. \\
\hline Infant immunisation & Proportion of newborns received BCG \\
\hline Waiting time (average) & Wait before seeing provider. \\
\hline Time spent with provider & Length of consultation. \\
\hline Baby or mother seen by provider & Within $48 \mathrm{~h}$, between 3-7 days, between 1-2 weeks' between 3-6 weeks, more than 6 weeks. \\
\hline Satisfaction & Satisfied, somewhat satisfied or not satisfied at all with services received. \\
\hline
\end{tabular}

midwives conducted consultations at voucher facilities (23.1\% versus $30.6 \% p=0.021)$.

Structural attributes of quality: supplies and commodities Overall there were no significant differences for availability of infrastructure, staffing, equipment, family planning commodities, medicines, and supplies in all facilities (Table 4). For the 111 features assessed, voucher facilities demonstrated $69.4 \%$ compared to $68.8 \%$ in non-voucher facilities. Additional analysis with a linear regression model shows non-significant scores, with voucher facilities scoring 2.4 points more than non-

Table 3 Characteristics of study facilities

\begin{tabular}{|c|c|c|c|c|}
\hline Key Features & Voucher facilities & Non-voucher facilities & Total & $P$ value \\
\hline Facility type & $n=21(\%)$ & $n=20(\%)$ & $n=41(\%)$ & \\
\hline Hospital & $15(71.4)$ & $12(60.0)$ & $27(65.8)$ & 0.162 \\
\hline Health centre & $4(19.0)$ & $8(40.0)$ & $12(29.2)$ & \\
\hline Nursing home & $2(9.5)$ & $0(0.0)$ & $2(4.8)$ & \\
\hline \multicolumn{5}{|l|}{ Sector } \\
\hline Public & $7(33.3)$ & $13(65.0)$ & $20(48.7)$ & 0.043 \\
\hline Private (NGO/Faith based) & $13(66.7)$ & $7(35.0)$ & $21(51.2)$ & \\
\hline \multicolumn{5}{|c|}{ Number of providers available and working in MCH/FP, Maternity unit, ART } \\
\hline Specialist doctors & 36 & 45 & 81 & Not significant \\
\hline Medical officers & 45 & 16 & 61 & \\
\hline Clinical officers & 46 & 50 & 96 & \\
\hline Registered nurses/midwifes & 204 & 146 & 350 & \\
\hline Enrolled nurses/midwives & 118 & 103 & 221 & \\
\hline Laboratory technologist /technicians & 11 & 24 & 35 & \\
\hline Pharmacists/technicians & 4 & 16 & 20 & \\
\hline Nutritionists & 20 & 16 & 36 & \\
\hline associated medical staff & 9 & 2 & 11 & \\
\hline Lay counsellors & 41 & 10 & 116 & \\
\hline Administrative staff & 12 & 21 & 33 & \\
\hline
\end{tabular}


Table 4 Basic infrastructure and provider training: structural attributes

\begin{tabular}{|c|c|c|c|c|}
\hline \multirow{2}{*}{$\begin{array}{l}\text { Mean score of facilities with the following equipment } \\
\text { /supplies (SD): }\end{array}$} & Voucher facilities $(n=21)$ & Non-voucher facilities $(n=20)$ & Total $(n=41)$ & $P$ value \\
\hline & $\mathrm{n} S \mathrm{SD}$ & $\mathrm{n} S \mathrm{SD}$ & \multicolumn{2}{|l|}{$\mathrm{n} S \mathrm{D}$} \\
\hline FP commodities (0-11) & $7.7(2.6)$ & $8.2(2.5)$ & $8.1(2.6)$ & 0.590 \\
\hline Testing reagents (0-12) & $10.0(1.9)$ & $9.9(2.1)$ & $9.5(2.1)$ & 0.814 \\
\hline General supplies (0-6) & $5.2(0.8)$ & $5.6(0.6)$ & $5.5(0.8)$ & 0.179 \\
\hline Essential vaccines (0-6) & $5.2(0.7)$ & $4.8(1.4)$ & $5.0(0.9)$ & 0.122 \\
\hline ARV Drugs (0-7) & $6.1(1.3)$ & $5.5(2.5)$ & $5.9(1.8)$ & 0.300 \\
\hline STI and RH drugs (0-10) & $4.2(3.7)$ & $4.6(3.5)$ & $5.3(3.6)$ & 0.719 \\
\hline Infection control supplies (0-9) & $7.2(1.3)$ & $7.1(1.3)$ & $6.7(1.4)$ & 0.655 \\
\hline FP supplies (0-18) & $14.8(3.9)$ & $14.6(3.5)$ & $14.2(3.6)$ & 0.891 \\
\hline Delivery supplies and kits (0-5) & $3.4(1.2)$ & $3.5(1.1)$ & $3.2(1.1)$ & 0.935 \\
\hline General equipment (0-6) & $5.9(0.3)$ & $5.7(0.6)$ & $5.7(0.5)$ & 0.323 \\
\hline Emergency equipment and drugs (0-4) & $3.0(1.1)$ & $3.3(0.8)$ & $3.0(0.9)$ & 0.329 \\
\hline General infrastructure (0-11) & $10.1(1.1)$ & $9.8(1.3)$ & $9.5(1.5)$ & 0.305 \\
\hline Total 0-111 (SD) & $83.3(13.4)$ & $82.6(12.1)$ & $82.9(12.6)$ & 0.855 \\
\hline \multicolumn{5}{|l|}{ Provider training and updates } \\
\hline \multirow[t]{2}{*}{$\%$ of providers receiving training in the last 24 months on: } & 90 & 73 & 163 & \multirow[t]{2}{*}{$P$ value } \\
\hline & $\mathrm{n} \%$ & n \% & $\mathrm{n} \%$ & \\
\hline Family planning & $26(28.9)$ & $19(26.0)$ & $45(27.6)$ & 0.684 \\
\hline Contraceptive technology update & $27(30.0)$ & $22(30.1)$ & 4930.1 & 0.985 \\
\hline Targeted PNC & $21(23.3)$ & $9(12.3)$ & $30(18.4)$ & 0.078 \\
\hline PMTCT & $37(41.1)$ & $30(41.1)$ & $67(41.1)$ & 0.998 \\
\hline Screening for cervical cancer & $28(31.1)$ & $12(16.4)$ & $40(24.5)$ & 0.030 \\
\hline Newborn care & $24(26.7)$ & $18(24.7)$ & $42(25.8)$ & 0.771 \\
\hline Essential obstetric care & $22(24.4)$ & $21(28.8)$ & $43(26.4)$ & 0.562 \\
\hline
\end{tabular}

${ }^{a} S D$ Standard deviation

voucher facilities (coefficient 2.49: $95 \%$ CI $(-5.29,10.28)$; $p=0.520$ ). Public voucher facilities had higher mean scores than non-voucher public facilities, 91.7 versus 83.2; $p=0.091$, with the regression model indicating a significant difference of 9.24 points higher than nonvoucher facilities (coefficient 9.24: $95 \%$ CI $(1.3,17.2) ; p$ $=0.024$, illustrated in Fig. 1 .

\section{Structural attributes of quality: provider knowledge and} reports on information they provide to postnatal women Providers said they received training on relevant maternal and newborn care, with no difference between facility groups (Table 4). Minimal overall differences were observed for providers' knowledge of postnatal care aspects in voucher and non-voucher facilities. While assessing reported provider practice, significantly more providers at voucher sites (than non-voucher facilities) reported counselling women on the importance of early initiation of breastfeeding (54.0 \% versus $38.4 \%: p=$ 0.048 ) during postnatal consultations. There were few differences between providers' reports of what maternal danger signs they advise postnatal women of, apart from significantly more voucher facility providers reported counselling on signs of severe pre-eclampsia ( $44.2 \%$ versus 26.0: $p=0.017$ ) in the immediate postnatal period (first 2 to 3 days after birth). Conversely, more providers at non-voucher facilities reported offering information on family planning to postnatal women $(83.6 \%$ versus $67 \%: p=0.017$ ).

\section{Process attributes of care: technical aspects}

Overall composite scores derived from our observations of consultations for postnatal care process attributes were generally low for both groups of facilities, with lower scores in voucher facilities, where 14.2 out of the 55 elements were demonstrated compared to 16.4 in non-voucher facilities, $p=0.0001$ (Table 5). Regression analysis indicates that voucher facilities scored $1.7 \%$ lower than non-voucher facilities, but this difference was not statistically significant; coefficient -1.70 (95\% CI) $(-4.9,1.5), p=0.294$ (Table 6$)$. There were also no significant differences for public facilities versus private facilities, nor for hospitals versus lower level facilities such as health centres and dispensaries. 


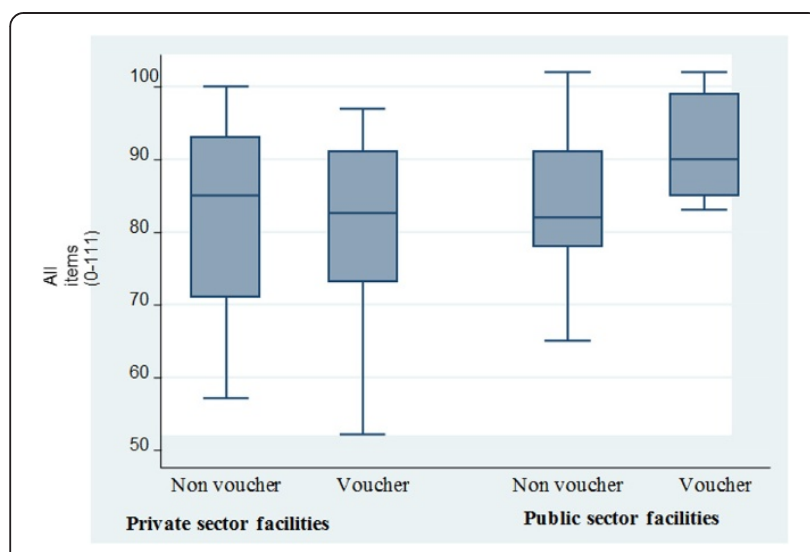

Fig. 1 Readiness score for structural attributes available (0-107): infrastructure, equipment, medicines commodities and supplies. Legend: Box plot showing summary scores for structural aspects of care (equipment, supplies, staffing, training) for private and public sector health facilities

For individual elements assessed in Table 5, nonvoucher facilities appear to have higher scores for history taking, fertility advice, infant feeding advice, and infant danger signs discussed, although these scores were not statistically significant when controlled for clustering. Public facilities did have 0.54 point higher scores for STI and HIV risk assessment compared to private facilities; coefficient. 0.27 (95\% CI) $(0.06,0.48) p=0.01$ (Table 6).

\section{Process attributes: interpersonal skills}

There were significant differences in average scores observed for rapport, with providers at voucher facilities scoring significantly lower than non-voucher facility providers (mean score 4.1 versus 4.4 out of $7: p=0.006$ ). There were individual variations on the seven aspects of building rapport, however. In voucher facilities more providers were observed using the client's name, but fewer were observed greeting the client or ensuring privacy or confidentiality. While comparing performances of public and private providers, those in public facilities scored 0.96 lower than private providers for building rapport during postnatal care consultations; coefficient $-0.96(95 \%$ CI $)(-1.61,0.32) p=0.004$ (Table 6).

\section{Outcome attributes}

Table 7 demonstrates some significant differences for waiting times of women at voucher and non-voucher facilities, but not for time spent with their provider. Women attending postnatal care at voucher facilities were more likely to have delivered in a health facility than women at non-voucher facilities (88.7 \% versus $77.8 \% ; p=0.001$ ) (data not shown in tables). More

Table 5 Observed provider practices (process attributes)

\begin{tabular}{|c|c|c|c|c|}
\hline \multicolumn{5}{|c|}{ Provider practices during postnatal care consultations (observed from client provider interactions) } \\
\hline \multirow[t]{2}{*}{ Mean scores for various constructs (SD) } & Voucher consultations $(n=479)$ & Non-voucher consultations $(n=241)$ & Total $(n=720)$ & \multirow{2}{*}{$\begin{array}{l}p \\
\text { values }\end{array}$} \\
\hline & SD & $\mathrm{SD}$ & SD & \\
\hline \multicolumn{5}{|l|}{ Maternal care } \\
\hline History taking practices $(0-7)$ & $2.2(1.7)$ & $2.7(1.8)$ & $2.4(1.7)$ & 0.0008 \\
\hline Physical examination of the mother $(0-7)$ & $1.1(1.8)$ & $1.3(1.9)$ & $1.2(1.8)$ & 0.214 \\
\hline Advice on danger signs for the mother (0-3) & $0.2(0.5)$ & $0.2(0.5)$ & $0.2(0.5)$ & 0.372 \\
\hline fertility advice (0-5) & $1.4(0.8)$ & $1.7(1.1)$ & $1.5(0.9)$ & 0.001 \\
\hline STI/HIV risk assessment (0-3) & $0.2(0.6)$ & $0.3(0.6)$ & $0.2(0.6)$ & 0.034 \\
\hline STI/HIV risk factors (0-4) & $0.1(0.6)$ & $0.2(0.5)$ & $0.2(0.6)$ & 0.779 \\
\hline STI management (0-3) & $0.02(0.2)$ & $0.02(0.1)$ & $0.1(0.2)$ & 0.812 \\
\hline Total for maternal care (0-32) & $4.6(4.3)$ & $5.8(4.4)$ & $5.0(4.4)$ & 0.0004 \\
\hline \multicolumn{5}{|l|}{ Infant care } \\
\hline Infant feeding advice (0-3) & $1.4(1.3)$ & $1.9(1.3)$ & $1.5(1.3)$ & $<0.001$ \\
\hline Infant examination $(0-4)$ & $1.0(0.9)$ & $0.9(0.8)$ & $0.9(0.9)$ & 0.275 \\
\hline Infant danger signs discussed (0-4) & $0.2(0.7)$ & $0.4(0.9)$ & $0.3(0.8)$ & 0.004 \\
\hline Total for infant care (0-11) & $2.6(2.1)$ & $3.2(2.1)$ & $2.8(2.1)$ & 0.002 \\
\hline \multicolumn{5}{|l|}{ Documentation } \\
\hline Total for documentation (0-4) & $2.9(1.1)$ & $2.9(1.3)$ & $2.9(1.2)$ & 0.796 \\
\hline \multicolumn{5}{|l|}{ Inter personal skills } \\
\hline Total creation of rapport (0-8) & $4.1(1.4)$ & $4.4(2.0)$ & $4.2(1.6)$ & 0.006 \\
\hline Total for process score (0-55) & $14.2(6.8)$ & $16.4(7.5)$ & $14.9(7.1)$ & 0.0001 \\
\hline
\end{tabular}


Table 6 Linear regression outputs for observed practices during postnatal care consultations

\begin{tabular}{|c|c|c|c|c|c|c|}
\hline \multirow{2}{*}{$\begin{array}{l}\text { Constructs } \\
\text { Maternal care }\end{array}$} & \multicolumn{6}{|c|}{ Coefficients for various construct scores $(95 \% \mathrm{Cl})$} \\
\hline & $\begin{array}{l}\text { Group (ref: voucher } \\
\text { facilities) }\end{array}$ & $\begin{array}{l}P \\
\text { value }\end{array}$ & $\begin{array}{l}\text { Sector (ref: public } \\
\text { sector) }\end{array}$ & $\begin{array}{l}P \\
\text { value }\end{array}$ & $\begin{array}{l}\text { Level of care: (ref: } \\
\text { hospital) }\end{array}$ & $p$ value \\
\hline History taking practices $(0-7)$ & $-0.25(-0.97,0.45)$ & 0.464 & $0.60(-0.13,1.32)$ & 0.103 & $-0.40(-1.0,0.21)$ & 0.191 \\
\hline Physical examination of the mother $(0-7)$ & $-0.10(-0.82,0.61)$ & 0.772 & $0.29(-0.44,1.0)$ & 0.427 & $0.16(-0.49,0.82)$ & 0.618 \\
\hline $\begin{array}{l}\text { Advice on danger signs for the mother } \\
(0-3)\end{array}$ & $-0.018(-0.22,0.18)$ & 0.857 & $0.081(-0.14,0.31)$ & 0.479 & $0.078(-0.09,0.25)$ & 0.369 \\
\hline fertility advice (0-5) & $-0.25(-0.69,0.17)$ & 0.239 & $0.54(0.16 .0 .92)$ & 0.006 & $0.11(-0.24,0.47)$ & 0.529 \\
\hline STI/HIV risk assessment (0-3) & $-0.02(-0.20,0.24)$ & 0.844 & $0.27(0.06,0.48)$ & 0.011 & $0.12(-0.04,0.29)$ & 0.152 \\
\hline STI/HIV risk factors (0-4) & $0.03(-0.19,0.25)$ & 0.783 & $0.16(-0.09,0.42)$ & 0.200 & $0.09(-0.10,0.28)$ & 0.346 \\
\hline STI management (0-3) & $0.013(-0.02,0.56)$ & 0.520 & $0.036(-0.009,0.083)$ & 0.115 & $0.023(-0.009,0.055)$ & 0.156 \\
\hline Total maternal care (0-32) & $-0.61(-2.6 .2 .37)$ & 0.535 & $2.00(-0.019,4.02)$ & 0.052 & $0.18(-1.45,1.83)$ & 0.817 \\
\hline \multicolumn{7}{|l|}{ Infant care } \\
\hline Infant feeding advice $(0-3)$ & $-0.40(-0.97,0.15)$ & 0.152 & $0.40(-0.17,0.99)$ & 0.167 & $0.31(-0.31,0.94)$ & 0.315 \\
\hline Infant examination (0-4) & $0.027(-0.27,0.32)$ & 0.854 & $-0.16(-0.51,0.17)$ & 0.327 & $-0.003(-0.31,0.30)$ & 0.980 \\
\hline Infant danger signs discussed (0-4) & $-0.12(-0.38,0.13)$ & 0.324 & $0.20(-0.004,0.42)$ & 0.055 & $0.10(-0.12,0.53)$ & 0.210 \\
\hline Total for infant care (0-11) & $-0.51(-1.29,0.27)$ & 0.198 & $0.44(-0.40,1.3)$ & 0.296 & $0.42(-0.52,1.33)$ & 0.365 \\
\hline Documentation (0-4) & $0.04(-0.48,0.56)$ & 0.877 & $0.048(-0.41,0.51)$ & 0.836 & $-0.01(-0.58,0.55)$ & 0.963 \\
\hline \multicolumn{7}{|l|}{ Interpersonal skills } \\
\hline Total creation of rapport (0-8) & $-0.62(-1.43,0.18)$ & 0.128 & $-0.96(-1.61,0.32)$ & 0.004 & $-0.50(-1.22,0.21)$ & 0.162 \\
\hline Total for process score $(0-55)$ & $-1.70(-4.9,1.5)$ & 0.294 & $1.53(-1.4,4.5)$ & 0.304 & $0.085(-2.8,3.0)$ & 0.954 \\
\hline
\end{tabular}

women (not significant) and newborns (significant $p<$ 0.001 ) at voucher facilities were seen within $48 \mathrm{~h}$ of childbirth (Table 7 ). Only $16 \%$ of women at voucher facilities and $17.7 \%$ at non-voucher facilities were interviewed leaving the maternity unit, which indicates a high proportion of women returning to facilities to access care within $48 \mathrm{~h}$ of birth. Similar proportions of women received their preferred family planning methods, and most received injectables or progestin-only pills.

Significantly more women at voucher facilities reported immunisation of their newborns with BCG during the postnatal care visit than at non-voucher facilities (82.5\% versus $76.5 \%$ : $p<0.001)$. Around $87 \%$ of women said they were satisfied with their treatment on the day of interview. Vouchers were utilised for antenatal care $(74.8 \%)$ and delivery services $(87.0 \%)$, but fewer than half were used for postnatal care $(48.5 \%)$.

\section{Discussion}

This analysis aims to understand the influence of Safe Motherhood vouchers on postnatal care quality by comparing public, private, and faith-based health facilities enrolled in the Kenya OBA program for four years (from 2006 to 2010) with a group of similar facilities with no access to vouchers. A quality of care framework adapted from Donabedian and Bruce grouped elements or attributes into structures, processes, and outcomes of postnatal care [32, 33]. Key indicators of interest include facilities' readiness to provide postnatal care (structures), maternal and infant counselling and care during a postnatal consultation (processes), and services women or their newborns actually received, including their perceptions of the care they received (outcomes).

Guidelines for postnatal care in Kenya are described in national documents dating from 2004, with a specific postnatal care register disseminated in 2005, and in 2007 an orientation package for targeted postnatal care was developed and distributed [34, 39]. In $201059 \%$ of Kenyan health facilities reported providing postnatal care [22] and accreditation criteria for facilities included provision of postnatal care. Our data show, however, that both voucher and non-voucher facilities scored below the expected scores for their overall postnatal care quality.

\section{Structure}

In OBA programs it is often assumed that their minimum quality standards for accreditation and the expected competition between health facilities encourage providers to improve their quality of care. Voucheraccredited public facilities do appear to score higher for structural attributes than non-voucher public facilities and all private facilities. In another component of the study, the evaluation team interviewed facility managers 
Table 7 Key outcome measures of quality of postnatal care (from exit interviews)

\begin{tabular}{|c|c|c|c|c|}
\hline \multirow[t]{2}{*}{$\%$ of clients who } & \multirow{2}{*}{$\begin{array}{l}\text { Voucher clients } \\
n(\%)\end{array}$} & \multirow{2}{*}{$\begin{array}{l}\text { Non-voucher clients } \\
n(\%)\end{array}$} & \multirow{2}{*}{$\begin{array}{l}\text { Total } \\
n(\%)\end{array}$} & \multirow[t]{2}{*}{$p$ value } \\
\hline & & & & \\
\hline Saw the provider within: & 468 & 244 & 712 & 0.081 \\
\hline within half hour of arriving at facility & $315(67.3)$ & $160(65.6)$ & $475(66.7)$ & \\
\hline 30 min-1 h & $37(7.9)$ & $13(5.3)$ & $50(7.0)$ & \\
\hline $1-2 \mathrm{~h}$ & $22(4.7)$ & $6(2.5)$ & $28(3.9)$ & \\
\hline above $2 \mathrm{~h}$ & $94(20.1)$ & $65(26.6)$ & $159(22.2)$ & \\
\hline Took the following time with provider & 468 & 244 & 712 & 0.674 \\
\hline $1-5 \min$ & $67(14.3)$ & $28(11.8)$ & $95(13.3)$ & \\
\hline $6-10 \mathrm{~min}$ & $103(22.1$ & $61(25.0)$ & $164(23.0)$ & \\
\hline $11-15 \min$ & $68(14.5)$ & $37(15.1)$ & $105(14.7)$ & \\
\hline 16-30 min & $68(14.5)$ & $40(16.3)$ & $108(15.2)$ & \\
\hline over half hour-3 $\mathrm{h}$ & $162(34.6)$ & 78 (31.9) & $240(33.7)$ & \\
\hline$\%$ of postnatal women who had undergone a first checkup: & 259 & 146 & 335 & 0.314 \\
\hline Within $48 \mathrm{~h}$ & $218(84.2)$ & $117(80.1)$ & $335(82.7)$ & \\
\hline Between 3 to 7 days & $5(1.9)$ & $2(1.3)$ & $7(1.7)$ & \\
\hline Between 1 to 2 weeks & $29(11.2)$ & $18(12.3)$ & $47(11.6)$ & \\
\hline Between 3 to 6 weeks & $6(2.3)$ & $9(6.2)$ & $15(3.7)$ & \\
\hline more than 6 weeks & $1(0.4)$ & $0(0.0)$ & $1(0.3)$ & \\
\hline Received preferred family planning method & $23(48.9)$ & $17(56.7)$ & $40(51.9)$ & 0.508 \\
\hline$\%$ of infants who had undergone a first checkup: & 426 & 229 & 655 & \\
\hline Within $48 \mathrm{~h}$ & $356(83.5)$ & $165(72.1)$ & $521(79.5)$ & 0.001 \\
\hline Between 3 to 7 days & $13(3.1)$ & $10(4.4)$ & $23(3.5)$ & \\
\hline Between 1 to 2 weeks & $43(10.4)$ & $34(14.8)$ & $77(11.8)$ & \\
\hline Between 3 to 6 weeks & $12(2.8)$ & $20(8.7)$ & $32(4.8)$ & \\
\hline More than 6 weeks & $2(0.4)$ & $0(0.0)$ & $2(0.3)$ & \\
\hline$\%$ reporting that they & 450 & 242 & 692 & 0.152 \\
\hline Satisfied with services & $399(88.7)$ & $208(85.9)$ & $607(87.7)$ & \\
\hline Somewhat satisfied with services & $349(7.5)$ & $28(11.5)$ & $62(8.9)$ & \\
\hline Not satisfied at all & $17(3.7)$ & $6(2.5)$ & $23(3.3)$ & \\
\hline
\end{tabular}

to ascertain how they utilised their voucher remittances [41]. Most used their funds on structural improvements such as renovating maternity units, laboratory or operating theatres as well as purchasing drugs, equipment or supplies. Moreover, public health facilities were required to follow strict $\mathrm{MoH}$ procedures for disbursed funds with formal requests to their county managers prior to any expenditures. Public facilities were also not able to use the funds for recruiting additional staff or on skills training $[41,42]$. This $\mathrm{MoH}$ policy may explain why public voucher facilities scored higher on structural attributes but lower on process [13, 41]. Slightly lower scores by private voucher facilities than private nonvoucher facilities may provide positive evidence that vouchers were correctly targeted at low income women who visit less sophisticated facilities.

Providers' knowledge and practice were also mixed. More voucher providers reported clients counselled on the importance of breastfeeding, but more non-voucher providers said they counselled women on family planning, which is unexpected due to the fact another voucher for family planning is available for poor women. From our review of facilities' remittances we know that little is spent on provider training [41], and we recommend that providers (both public and private) have more access to training sessions and contraceptive technology updates organised by the Ministry of Health.

Although these high structural attribute scores could be taken as synonymous with availability or readiness to 
provide services and commodities, they are incomplete, because clinical or process attributes for the provision (and receipt) of comprehensive postnatal care are not included [32]. A 2010 review and design of an extension to the OBA programme also concluded that postnatal care had yet to change significantly by virtue of reimbursement funds alone [42].

\section{Process}

All facilities' composite scores totalled less than one third of the maximum score, well below the standards of postnatal care provision in the national guidelines [34, 43]. Although voucher facilities scored significantly worse than non-voucher facilities, no significant differences overall were observed for public and private facilities or hospitals and lower level facilities. Non-voucher facilities performed better in history taking and counselling on fertility and infant care, but these scores were not significant when controlled for clustering. Public facilities scored higher on counselling for HIV and STI risk assessment and family planning than private facilities, but private facilities had higher scores for rapport.

The overall low performance in clinical care (or process attributes) at both voucher and non-voucher sites could be explained by several factors. Although providers reported some clinical updates for a range of maternal and newborn care, it is possible providers were unable to translate this knowledge into practice [44]. Secondly, although women are entitled to postnatal care with the Safe Motherhood voucher, providers often only immunise an infant rather than providing a comprehensive package of care for both mother and child. The difficulty of conducting a check up for a mother in a busy immunisation clinic, without privacy or examination couches, can contribute to infant-focused care. Thirdly, women may not be aware of the comprehensive package of postnatal care, as over $60 \%$ of women in this study came to postnatal care for infant immunisation. Elsewhere in the Kenya voucher evaluation we found communities with misunderstanding about what services vouchers actually cover [26]. Finally, although there are now separate vouchers for each of the four antenatal care visits as well as childbirth, there is no specific paper voucher for postnatal care, so facilities can only claim reimbursement for this service as an extension of the delivery voucher (i.e., if a woman give birth in a facility), but women are only encouraged to return to a facility after six weeks for infant immunisation. Nevertheless, it was expected that the OBA programme would improve the overall quality of maternal care including postnatal care.

If voucher facilities are not reimbursed for providing comprehensive postnatal care, this may hinder providers' motivation for offering any postnatal services for mothers and infants, especially in private facilities that do not receive updates from $\mathrm{MoH}$. There are clearly defined guidelines and standards about the content and timing of mother and infant care immediately after birth, at one or two weeks postpartum, and up to six weeks after birth. Many providers, specifically those from private facilities, appear to be unaware of these national postnatal guidelines, and this contributes to service discontinuity after pregnancy and delivery $[3,45]$.

\section{Outcomes}

More than four fifths of all women were seen within $48 \mathrm{~h}$, and significantly more newborns at voucher facilities received care in their first $48 \mathrm{~h}$ in addition to BCG vaccinations than those at non-voucher facilities. Evidence suggests that newborns seen within the first two days of birth by a provider have a greater chance of survival [46]. Overall, postnatal women seemed satisfied with their care, waiting times, and time spent with a provider, regardless of facility type. Clinical competence is less easily judged by clients, and often they evaluate providers on the amount of time spent with them, and their caring attitude, than on technical skills [32]. Although the output attributes based on clients' experience may not necessarily translate into adequate clinical care, such information presents an opportunity for the health system to understand women's perceptions of quality postnatal care and what motivates them to seek services.

A comprehensive postnatal care package should include routine visits in the days following childbirth, when risks are high for both mother and baby, complemented by promotion of healthy behaviours (e.g., exclusive breast feeding), for identifying complications and facilitating referrals [3]. Kenya's $\mathrm{MoH}$ recommends one visit within $48 \mathrm{~h}$ of delivery, another within one week, a third between four and six weeks postpartum, and a fourth at six months [39]. Postnatal registers have been in existence in Kenya since 2005, but few facilities routinely record or collect this information, or report it through the government health information system. An attempt to deduce potential increased workloads associated with increased in attendance at voucher facilities and its effect on quality of care was not possible due to these recording gaps. The OBA programme's voucher management agency is in the process of converting the paper vouchers to a 'smart card' that may capture all services offered to and received by voucher clients, including postnatal care.

A review of the literature on the quality of private and public health care in low and middle income countries indicates that improving quality of care in a health system is a long term effort and requires attention to various aspects including the incentive structure and providers' training [47]. Supervision and clinical audits 
with resultant recommendations, especially if combined with training, have been found to be effective in improving quality [48]. The OBA project itself did not provide any technical training, and expected health facilities to improve their own staff skills through technical updates. Apparently this did not happen sufficiently, although providers received some related maternal and infant care training within the past 24 months.

For the adequate clinical care of postnatal women, and the diagnosis and early treatment of any complications, an evidence-based schedule for visits is critical. While Kenya has detailed the timing and content for postnatal visits in policy guidelines, it appears there is a policy and care provision gap: Many health care providers do not perceive the importance of the postnatal period, although four fifths of women interviewed sought care within $48 \mathrm{~h}$ postpartum. A community-based approach may be required to ensure that women discharged after delivery receive adequate information, support, and follow up. Providers should receive more training updates specifically for the postnatal period, and any training should be followed by supportive supervision. Policy guidelines should be more easily available for private and faith-based facilities.

In other results-based financing initiatives, supply side incentives reward improvements in structural and process indicators for quality. These programs develop verification mechanisms that routinely confirm service deliveries and measure quality indicators at contracted facilities. Most OBA voucher programs do not have an explicit financial incentive for quality improvements, and it might be worthwhile for the Kenya program to consider one [18].

There are limitations to this analysis. Its cross sectional design, coupled with a non-random facility selection, limits any tests for causality. Control facilities were, however, matched by administrative type and similar infrastructure, staffing and service characteristics, along with a qualitative assessment of each local healthcare market and access. Ideally, the voucher and non-voucher facilities would be alike in every way except for the OBA voucher contract. Because facilities differed according to ownership status it is possible other differing factors, such as financing and catchment size, could affect quality of care outcomes. Secondly, although comparison sites were carefully selected, support by other partners for maternal and newborn health may also have confounded results. We did control for clustering at facility level, provider type, and level of care. Thirdly, the study population of postnatal women attending a health facility is not representative of Kenya's general postnatal population, where only $44 \%$ of newly delivered women attend postnatal care [20]. These findings do, however, represent the services received by recently delivered women in most Kenyan health facilities.

\section{Conclusion}

Overall, the quality of postnatal care in all facilities was low, which indicates that the postnatal period continues to receive limited attention from both women and providers, even where a Safe Motherhood voucher exists. The OBA voucher programme should include separate reimbursement for comprehensive postnatal care services to address this critical period. In addition, the voucher programme should explore incentives for quality improvement targets in each service subsidised by the programme.

\section{Abbreviations}

ANC: Antenatal Care; BCG: Bacillus Calmette-Guerin; CPI: Client-Provider Interaction; HFA: Health Facility Assessment; IRB: Institutional Review Board; KEMRI: Kenya Medical Research Institute; KfW: German Development Bank; MNH: Maternal and Newborn Health; MoH: Ministry of Health; PNC: Postnatal Care; QoC: Quality of Care; OBA: Output-based Aid; SM: Safe Motherhood; STI: Sexually Transmitted Infection; VMA: Voucher Management Agency.

\section{Competing interests}

The authors declare that they have no competing interests.

\section{Authors' contributions}

CEW was responsible for drafting the manuscript and in development of QoC framework. CEW and TA were involved in analysis of data. CEW and BB were substantially involved in conceptualizing the study design. TA, LK, FO, RN, MT and BB in reviewing the manuscript for intellectual content. All authors read and approved the final manuscript.

\section{Acknowledgements}

This work was supported by the Bill and Melinda Gates Foundation as part of a multi country study evaluation of voucher-and-accreditation interventions. Grant number 51761

\section{Author details}

'Population Council, 4301 Connecticut Avenue NW, Washington DC 20008, USA. ${ }^{2}$ Population Council General Accident Insurance House, Ralph Bunche Road, PO Box 17643, Nairobi 00500, Kenya. ${ }^{3}$ Department of Obstetrics and Gynecology, Faculty of Medicine and Health Sciences, Ghent University, De Pinte Laan 185, B-9000 Ghent, Belgium.

Received: 10 March 2015 Accepted: 13 July 2015

Published online: 24 July 2015

\section{References}

1. Lawn JE, Cousens S, Zupan J. 4 million neonatal deaths: when? Where? Why? Lancet. 2005;365(9462):891-900.

2. Save the Children. Surviving the First Day: State of the World's Mothers. London: Save the Children; 2013.

3. Kerber K, de Graft-Johnson JE, Buttha ZA, Okong P, Starrs A, Lawn JE. Continuum of care for maternal, newborn and child health: from slogan to service delivery. Lancet. 2007;370:1358-68.

4. WHO. Technical Consultation on Postpartum and Postnatal Care. Geneva: Department of Making Pregnacy Safer, WHO; 2010.

5. Kinney M, Kerber K, Black RE, Cohen B, Nkrumah F, Coovadia H, et al. SubSaharan Africa's Mothers, Newborns, and Children: Where and Why Do They Die? PLoS Med. 2010;7(6):e1000294.

6. Ir P, Horemans D, Souk N, VanDamme W. Using targeted vouchers and health equity funds to improve access to skilled birth attendants for poor women: a case study in three rural health districts in Cambodia. BMC Pregnancy Childbirth. 2010;10:1

7. Gabrysch S, Campbell OM. Still too far to walk: literature review of the determinants of delivery service use. BMC Pregnancy Childbirth. 2009;9:34. 
8. Kruk M, Galea S, Prescott M, Freedman LP. Health care financing and utilization of maternal health services in developing countries. Health Policy Plan. 2007;22(5):303-10.

9. Sekabaraga C, Diop F, Soucat A. Can innovative health financing policies increase access to MDG-related services? Evidence from Rwanda. Health Policy Plan. 2011;26 Suppl 2:ii52-62.

10. Obare F, Warren C, Abuya T, Askew I, Bellows B. Assessing the populationlevel impact of vouchers on access to health facility delivery for women in Kenya. Soc Sci Med. 2014;102:183-9.

11. Obare F, Warren CE, Njuki R, Abuya T, Sunday J, Askew I, et al. Communitylevel impact of the reproductive health vouchers programme on service utilization in Kenya. Health Policy Plan. 2013;28(2):165-75.

12. Ensor T. Consumer-led demand side financing in health and education and its relevance for low and middle income countries. Int J Health Plann Manage. 2004;19(3):267-85.

13. Abuya T, Njuki R, Warren CE, Okal J, Obare F, Kanya L, et al. A policy analysis of the implementation of a Reproductive Health Vouchers Program in Kenya. BMC Public Health. 2012;12:540.

14. Ahmed S, Khan MM. A maternal health voucher scheme: what have we learned from the demand-side financing scheme in Bangladesh? Health Policy Plan. 2011;26(1):25-32.

15. Murray S, Hunter BM, Bisht R, Ensor T, Bick D. Effects of demand-side financing on utilisation, experiences and outcomes of maternity care in low- and middle-income countries: a systematic review. BMC Pregnancy Childbirth. 2014;14:30.

16. Schmidt J, Ensor T, Hossain A, Khan S. Vouchers as demand side financing instruments for health care: a review of the Bangladesh maternal voucher scheme. Health Policy. 2011;96:98-107.

17. Gupta I, Joe W, Rudra S. Demand Side Financing in Health: How far can it address the issue of low utilization in developing countries? In: World Health Report. Geneva: World Health Organisation; 2010. p. 27.

18. Bonfrer I, Soeters R, Van de Poel E, Basenya O, Longin G, van de Looij F, et al. Introduction of performance-based financing in Burundi was associated with improvements in care and quality. Health Aff (Millwood) 2014;33(12):2179-87.

19. Soeters R, Habineza C, Peerenboom PB. Performance-based financing and changing the district health system: experience from Rwanda. Bull World Health Organ. 2006;84(11):884-9.

20. Kenya National Bureau of Statistics (KNBS). Kenya Demographic and Health Survey 2008-09. Calverton: KNBS and ICF Macro; 2010.

21. Fort A, Kothari T, Abderrahim N. Postpartum Care: Levels and Determinants in Developing Countries. In: DHS Comparative Reports 15. Calverton: Macro International Inc; 2006

22. NCAPD, MOMS, MPHS, KNBS, ICF Macro. Kenya Service Provision Assessment Survey 2010. Nairobi: National Coordinating Agency for Population and Development, Ministry of Medical Services, Ministry of Public Health and Sanitation, Kenya National Bureau of Statistics, and ICF Macro; 2011.

23. Hagenmeyer E, Griffin D, Ahmed R, Teshome A, Barsch A, Muga R, et al. Design study for output-based assistance programme Kenya: Final report. Berlin: IGES Paper 05-67; 2005.

24. PriceWaterhouse Coopers. Reproductive health output-based aid: End of pilot project report. Vol. 1. Nairobi: Pricewaterhouse Coopers; 2008.

25. RH-OBA Technical Committee. RH-OBA Review on Quality Assurance: Final Report. Nairobi: RH-OBA Technical Committee; 2009.

26. Njuki R, Obare F, Warren C, Abuya T, Okal J, Mukuna W, et al. Community experiences and perceptions of reproductive health vouchers in Kenya. BMC Public Health. 2013;13:660.

27. IGES. Creating and Efficient and Quality Health Care System. Output based Aid 2015; Available from: www.output-based-aid.net.

28. Njuki R, Okal J, Warren CE, Obare F, Abuya T, Kanya T, et al. Exploring the effectiveness of the output-based aid voucher program to increase uptake of gender-based violence recovery services in Kenya: A qualitative evaluation. BMC Public Health. 2012;12:426.

29. Brody C, Bellows N, Campbell M, Potts M. The impact of vouchers on the use and quality of health care in developing countries: a systematic review Glob Public Health. 2013;8(4):363-88.

30. Bellows N, Bellows BW, Warren C. Systematic Review: the use of vouchers for reproductive health services in developing countries: systematic review. Trop Med Int Health. 2011;16(1):84-96.

31. Warren C, Abuya T, Obare F, Sunday J, Njue R, Askew I, et al. Evaluation of the impact of the voucher and accreditation approach on improving reproductive health behaviors and status in Kenya. BMC Public Health. 2011;11:177.

32. Bruce J. Fundamental Elements of Quality of Care: A Simple Framework. Stud Fam Plan. 1990;21(2):61-91.

33. Donabedian A. The quality of care. How can it be assessed? JAMA 1988;260(12):1743-8

34. Ministry of Health. National Guidelines for Quality Obstetrics and Perinatal Care. Nairobi: Ministry of Health; 2004

35. Ministry of Health. The Second National Health Sector Strategic Plan of Kenya (NHSSP II 2005-10). Nairobi: Ministry of Health; 2005.

36. Premier I, CMS HQI Demonstration Project. Composite Quality Score Methodology Overview. [cited 2014; Available from: https:// www.premierinc.com/quality-safety/tools-services/p4p/hqi/resources/ composite-scoring-overview.pdf.

37. Delvaux T, Konan JP, Ake-Tano O, Gohou-Kouassi V, Bosso PE, Buve A, et al, Quality of antenatal and delivery care before and after the implementation of a prevention of mother-to-child HIV transmission programme in Cote d'Ivoire. Trop Med Int Health. 2008;13(8):970-9.

38. Kyei N, Chansa C, Gabrysch S. Quality of antenatal care in Zambia: a national assessment. BMC Pregnancy Childbirth. 2012;12:151.

39. Ministry of Health Kenya. Orientation Package for Targeted Postnatal Care. Nairobi: Division of Reproductive Health; 2007.

40. WHO. Make Every Mother and Child Count. In: The World Health Report. Geneva: World Health Organization; 2005.

41. Abuya T, Sanghrajka S, Warren CE, Obare F, Kanya L, Askew I, et al. Health Facility Spending Patterns of Reproductive Health Voucher Reimbursement Revenue among accredited facilities in Kenya. In: RH Vouchers Project Working Paper. Nairobi: Population Council; 2013.

42. Gorter A, Bayou A. Midterm review: Kenya development of the health sector (SWAp) programme reproductive health voucher scheme (output based approach) project. Bad Homburg: EPOS Health Management; 2011.

43. Ministry of Health. National Guidelines for Quality Obstetrics and Perinatal Care. Nairobi: D.o.R. Health. 2011/2012

44. Boller C, Wyss K, Mtasiwa D, Tanner M. Quality and comparison of antenatal care in public and private providers in the United Republic of Tanzania. Bull World Health Organ. 2003;81(2):116-22.

45. WHO. WHO recommendations on postnatal care of the mother and newborn. 2014.

46. Moran AC, Kerber K, Sitrin D, Guenther T, Morrissey CS, Newby H, et al. Measuring coverage in $\mathrm{MNCH}$ : indicators for global tracking of newborn care. PLoS Med. 2013;10(5), e1001415

47. Berendes S, Heywood P, Oliver S, Garner P. Quality of private and public ambulatory health care in low and middle income countries: systematic review of comparative studies. PLoS Med. 2011;8(4), e1000433.

48. Rowe AK, de Savigny D, Lanata CF, Victora CG. How can we achieve and maintain high-quality performance of health workers in low-resource settings? Lancet. 2005;366(9490):1026-35.

\section{Submit your next manuscript to BioMed Central and take full advantage of:}

- Convenient online submission

- Thorough peer review

- No space constraints or color figure charges

- Immediate publication on acceptance

- Inclusion in PubMed, CAS, Scopus and Google Scholar

- Research which is freely available for redistribution 